Recepción: 01 / 03/ 2018

Aceptación: 15 / 05 / 2018

Publicación: 07 / 07 / 2018

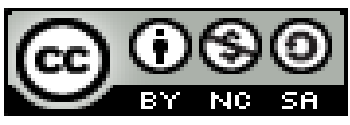

Ciencias de la Salud

Artículo de Investigación

\title{
Responsabilidad Civil y Penal de la práctica de enfermería en Ecuador
}

\author{
Civil and Criminal Liability of Nursing Practice in Ecuador

\section{Responsabilidade Civil e Penal da Prática de Enfermagem no Equador}

Sidar E. Solórzano-Solórzano I

sidarsolorzano@hotmail.com

Delia G. Bravo-Bonoso II

deliabravo85@hotmail.com

Estrella M. Mero-Quijije ${ }^{\text {III }}$

estrella.mero@hotmail.com

\author{
María E. Pincay-Cañarte ${ }^{\text {IV }}$ \\ maría.pincay@hotmail.com \\ Dolores M. Cedeño-Holguín V \\ dolores.cedeno@hotmail.com
}

Correspondencia: sidarsolorzano@hotmail.com

I. Magister en Gerencia Educativa; Licenciada en Ciencias de la Educación Especialidad Castellano y Literatura; Abogado de los Juzgados y Tribunales de la Republica, Universidad Estatal del Sur de

II Manabí Jipijapa, Ecuador.

II. Magister en Emergencias Médicas; Licenciada en Ciencias de la Enfermería, Universidad Estatal del Sur de Manabí Jipijapa, Ecuador.

III. Magister en Gerencia en Salud para el Desarrollo Local; Licenciada en Ciencias de la Enfermería, Universidad Estatal del Sur de Manabí Jipijapa, Ecuador.

IV. Magister en Gerencia en Salud para el Desarrollo Local; Licenciada en Enfermería, Universidad Estatal del Sur de Manabí Jipijapa, Ecuador.

V. Magister en Gerencia en Salud para el Desarrollo Local; Licenciada en Ciencias de la Enfermería, Universidad Estatal del Sur de Manabí Jipijapa, Ecuador. 
Sidar E. Solórzano-Solórzano; Delia G. Bravo-Bonoso; Estrella M. Mero-Quijije; María E. Pincay-Cañarte; Dolores M.

\section{Resumen}

El ejercicio de la enfermería como toda práctica profesional implica una serie de responsabilidades de tipo ético y legal, en ese sentido, los Organismos Internacionales y Nacionales de Salud han regulado dichas áreas a los fines de mantener una equidad entre el ejercicio de la profesión y el paciente, así como delimitar sus obligaciones y responsabilidades. El presente trabajo estudia las responsabilidades civiles y penales de los profesionales de la Enfermería en Ecuador. La metodología usada se basó en un estudio de tipo documental informativa en donde se recopiló una serie de documentos electrónicos e impresos para analizar el tema en cuestión. La responsabilidad tanto civil como penal viene dada por la mala práctica en el ejercicio de la profesión de la enfermería. Los delitos por mala práctica profesional, así como su sanción o pena, en Ecuador se encuentran establecidos en el Código Civil y la Ley Orgánica Integral Penal. Es importante para el enfermero y/o enfermera conocer las regulaciones en estas materias en principio para garantizar la idoneidad del ejercicio de la profesión con el objeto de evitar demandas y al mismo tiempo ayudar a proteger los derechos de los pacientes.

Palabras claves: Responsabilidad, Civil, Penal, Práctica, Enfermería. 


\section{Abstract}

The practice of nursing as any professional practice implies a series of ethical and legal responsibilities, in this sense, the International and National Health Organizations have regulated these areas in order to maintain a fair balance between the exercise of the profession and the patient, as well as define their obligations and responsibilities. This paper studies the civil and criminal responsibilities of Nursing professionals in Ecuador. The methodology used was based on an informative documentary type study where a series of electronic and printed documents were collected to analyze the subject in question. The responsibility, both civil and criminal, is given by the bad practice in the exercise of the profession of nursing. The crimes for professional malpractice, as well as its sanction or punishment, in Ecuador are established in the Civil Code and the Comprehensive Organic Penal Law. It is important for the nurse and / or nurse to know the regulations in these matters in principle to ensure the suitability of the profession in order to avoid lawsuits and at the same time help protect the rights of patients.

Keywords: Responsibility, Civil, Criminal, Practice, Nursing. 


\section{Introducción.}

Las normas son reglas que han existido desde el principio de los tiempos, ellas nos dicen qué hacer o cómo hacerlo, persiguiendo el bienestar común. Existen muchos tipos de normas: normas religiosas, normas morales, normas éticas, normas sociales, normas jurídicas, entre otras.

Las prácticas profesionales no escapan estas regulaciones, en el presente caso estudiaremos las normas jurídicas relacionadas con la enfermería, específicamente estableceremos cuáles son las leyes vigentes que regulan la práctica de la enfermería en la República del Ecuador y los artículos relacionados con la responsabilidad tanto civil como penal, a los fines de resaltar la importancia que tiene el conocimiento de dicha legislación para todos los ciudadanos ecuatorianos, en especial los enfermeros y enfermeras.

En tal sentido, podemos decir que la responsabilidad es una obligación de hacer o no hacer, cuyo incumplimiento, en derecho, acarrea una sanción o pena, dependiendo del caso.

Por otra parte, se define la responsabilidad civil como: "la obligación de un sujeto de reparar, a través de una compensación monetaria, un daño que le haya provocado a otra. Lo habitual es que la responsabilidad civil obligue al responsable del daño a pagar una indemnización por los perjuicios que ocasionó”. (Definición, s.f.)

La responsabilidad penal por su parte, se refiere al: "deber jurídico que se impone a un individuo imputable de responder de su acción antijurídica prevista en el Código Penal como delito, de la que es culpable, debiendo sufrir sus consecuencias jurídicas.” (Wolter Skluwer, s.f.) 
Según (Carrara, 1991) el delito es la infracción a la Ley del Estado, promulgada para proteger la seguridad de los ciudadanos y que resulta de un acto externo del hombre, positivo o negativo, moralmente imputable y políticamente dañoso. (p. 43)

El delito puede ser de dos tipos: Doloso y Culposo. Para (Jiménez de Asúa, 1997) existe dolo cuando:

Se produce un resultado típicamente antijurídico, con conciencia de que se quebranta el deber, con conocimiento de las circunstancias de hecho y del curso esencial de la relación de causalidad existente entre la manifestación humana y el cambio en el mundo exterior, con voluntad de realizar la acción y con representación del resultado que se quiere o ratifica. (p. 243)

Mientras que Culpa es el término jurídico que, según Carrara, citado por (Grisanti, 2005), al igual que la negligencia, supone la "voluntaria omisión de diligencia en calcular las consecuencias posibles y previsibles del propio hecho“.

Es importante definir los tipos de delito, ya que en materia penal de ello va a depender su calificación. El dolo y la culpa inciden directamente en la cuantía de la pena.

Ciertamente, la responsabilidad del profesional de enfermería se traduce en los deberes y obligaciones que este debe cumplir, y en asumir las consecuencias de sus acciones, en tal sentido, la responsabilidad legal viene dada principalmente por la mala práctica de estos deberes y obligaciones y los resultados negativos que trasgreden la ley y ocasionan un perjuicio. En virtud de lo cual, surge la responsabilidad como la imposición (al que trasgrede la norma) por parte del Estado de asumir las consecuencias de sus actos, en este caso, jurídicas, en materia civil se traduce al resarcimiento 
Sidar E. Solórzano-Solórzano; Delia G. Bravo-Bonoso; Estrella M. Mero-Quijije; María E. Pincay-Cañarte; Dolores M.

pecuniario de daños y en materia penal al cumplimiento de una pena, en ambos casos previamente o acompañado de sanción de un delito.

\section{Metodología.}

La investigación documental o diseño documental "es un proceso basado en la búsqueda, recuperación, análisis, critica e interpretación de datos secundarios, es decir los obtenidos y registrados por otros investigadores en fuentes documentales: impresas, audiovisuales o electrónicas”. (Arias, 2012)

En tal sentido, el presente estudio se realizó mediante una investigación de tipo documental, proceso en el cual se desarrolló una búsqueda de información mediante un computador con conexión a internet, a través de los buscadores Google Académico ${ }^{\circledR}$ y Google $^{\circledR}$ en donde se recopilaron fuentes documentales electrónicas relacionadas con el tema en estudio, asimismo, se recopilaron y revisaron fuentes impresas, para posteriormente realizar un análisis crítico del mismo. En el presente estudio no se trata de aprobar o no posturas, la información aquí presente se basa en lo encontrado en las fuentes, se trata de un análisis relacionado con la responsabilidad civil y penal de la práctica de la enfermería contenida en los instrumentos legales de Ecuador.

\section{Resultados.}

Es importante destacar que para determinar la configuración de un delito ocasionado en el ejercicio de la función de la enfermería, se debe establecer el campo de acción de este profesional, sus deberes y obligaciones. 
Al respecto, la (Organización Mundial de la Salud, 1996) indica:

Cuestiones tales como la esfera de acción de la enfermería, la enseñanza de la enfermería y el funcionamiento del sistema de asistencia sanitaria están regidas por la ley. La legislación puede, por lo tanto, asistir u obstaculizar el desarrollo del ejercicio de la enfermería y su efectiva contribución al sistema de asistencia sanitaria. Como la legislación aplica las normas, su formulación deberá tener en cuenta las normas generales para el desarrollo de recursos humanos para la salud y ligada a ellas. (p. 17)

En este sentido, la (Ley del Ejercicio Profesional de las Enfermeras y Enfermeros del Ecuador, 1998) ampara y garantiza el ejercicio profesional de la enfermería, enmarca competencias, deberes y obligaciones, que son fundamentales para establecer la configuración o no de un delito.

Igualmente, la (Ley Orgánica de Salud, 2012) enmarca a nivel general, para el profesional o personal de salud los deberes y obligaciones, dicha Ley se enfoca en el procedimiento administrativo en caso de infracción, sin perjuicio de las responsabilidades civiles y/o penales que se deriven del incumplimiento de la norma.

Partiendo de la concepción de que la responsabilidad en la práctica de la enfermería viene dada por una serie de normativas que delimitan el deber ser y actuar de este profesional y que su incumplimiento conlleva a sanciones o penas, según sea el caso, vamos a puntualizar dentro de la legislación de la República del Ecuador las principales normas civiles y penales con sus respectivos artículos relacionados con las malas prácticas en el ejercicio de esta profesión.

La (Constitución de la República del Ecuador, 2008), en su artículo 54, inciso segundo, establece: 
Sidar E. Solórzano-Solórzano; Delia G. Bravo-Bonoso; Estrella M. Mero-Quijije; María E. Pincay-Cañarte; Dolores M. Cedeño-Holguín

Las personas serán responsables por la mala práctica en el ejercicio de su profesión, arte u oficio, en especial aquella que ponga en riesgo la integridad o la vida de las personas.

\section{Responsabilidad Penal}

Se encuentra establecida en el Código Orgánico Integral Penal publicado por el (Ministerio de Justicia, Derechos Humanos y Cultos de la República del Ecuador, 2014) en su artículo 146:

La persona que al infringir un deber objetivo de cuidado, en el ejercicio o práctica de su profesión, ocasione la muerte de otra, será sancionada con pena privativa de libertad de uno a tres años.

El proceso de habilitación para volver a ejercer la profesión, luego de cumplida la pena, será determinado por la Ley.

Será sancionada con pena privativa de libertad de tres a cinco años si la muerte se produce por acciones innecesarias, peligrosas e ilegítimas.

Para la determinación de la infracción al deber objetivo de cuidado deberá concurrir lo siguiente: 1. La mera producción del resultado no configura infracción al deber objetivo de cuidado. 2. La inobservancia de leyes, reglamentos, ordenanzas, manuales, reglas técnicas o lex artis aplicables a la profesión. 3. El resultado dañoso debe provenir directamente de la infracción al deber objetivo de cuidado y no de otras circunstancias independientes o conexas. 4. Se analizará en cada caso la diligencia, el grado de formación profesional, las condiciones objetivas, la previsibilidad y evitabilidad del hecho. (p. 70) 
Con relación a dicha disposición legal se emite la Resolución No. 01-2014 Corte Nacional de Justicia, contenida en el mismo libro del (Ministerio de Justicia, Derechos Humanos y Cultos de la República del Ecuador, 2014) y en atención a la solicitud de aclaratoria solicitada por La Comisión que representa a diversas Federaciones de Médicos, Enfermeras, Odontólogos, y Obstetrices; Colegios profesionales del sector de la salud, Hospitales, y Sociedades Científicas, donde establecen en base al alcance del mencionado artículo:

Que la norma transcrita, en el inciso primero tipifica el homicidio culposo simple por mala práctica profesional, el que se configura por la infracción al deber objetivo de cuidado conforme al inciso final del artículo 146 del Código Orgánico Integral Penal; en tanto que, el inciso tercero tipifica la figura de homicidio culposo calificado o agravado, en el que, a más de la infracción al deber objetivo de cuidado, deben concurrir "acciones innecesarias, peligrosas e ilegítimas.

\section{Al respecto, Resuelve:}

- Art. 1.- El Código Orgánico Integral Penal, que en su artículo 146, establece los tipos penales simple y calificado de homicidio culposo por mala práctica profesional, debe ser comprendido en su integridad.

- Art. 2.- Se entenderá que el homicidio culposo simple por mala práctica profesional, tipificado en el inciso primero del artículo 146 del Código Orgánico Integral Penal, se configura por la inobservancia del deber objetivo de cuidado, conforme a su inciso final.

- Art. 3.- Se entenderá que el homicidio culposo calificado por mala práctica profesional, tipificado en el inciso tercero del artículo 146 del Código Orgánico Integral Penal, se configura por la inobservancia del deber objetivo de cuidado; y, además, por la concurrencia de las acciones innecesarias, peligrosas e ilegítimas. 
Sidar E. Solórzano-Solórzano; Delia G. Bravo-Bonoso; Estrella M. Mero-Quijije; María E. Pincay-Cañarte; Dolores M. Cedeño-Holguín

- Disposición Final.- Esta resolución entrará en vigencia con el Código Orgánico Integral Penal. (325)

El (Ministerio de Salud Pública de la República del Ecuador, 2014) explica acerca de las cuatro condiciones que deben cumplirse para que se apertura la posibilidad del incumplimiento del deber objetivo de cuidado que conforma el artículo 146 del Código Orgánico Integral Penal:

Con respecto a que la mera producción del resultado no configura infracción al deber objetivo de cuidado, se refiere a que la muerte de un paciente no significa que necesariamente el médico haya infringido al deber objetivo del cuidado. Se analizará el desarrollo de los hechos que concluyeron en el fallecimiento del paciente y no solo el resultado. Con relación a la inobservancia de leyes, reglamentos, ordenanzas, manuales, reglas técnicas o lex artis aplicables a la profesión, significa que el no apego a los estándares básicos de la profesión, es una de las condiciones que debe concurrir con todas las otras para que se configure la infracción del deber objetivo de cuidado, pero no la única. En cuanto a que el resultado dañoso debe provenir directamente de la infracción al deber objetivo de cuidado y no de otras circunstancias independientes o conexas, quiere decir que si un paciente fallece por falta de insumos o medicamentos, infraestructura inadecuada, causas propias de la enfermedad o por características propias de la persona, no es responsabilidad del médico tratante. Por último, en cuanto al análisis en cada caso la diligencia, el grado de formación profesional, las condiciones objetivas, la previsibilidad y evitabilidad del hecho, esto es que cada caso implica un análisis técnico, un proceso que precisa de la concurrencia de peritos especializados en función de la naturaleza del caso. 


\section{Responsabilidad Civil}

Se encuentra, de forma general, representada en el (Código Civil de la República del Ecuador, 2016) en cuanto a la responsabilidad civil de la comisión de un delito o cuasi delito, establece:

El que ha cometido un delito o cuasidelito que ha inferido daño a otro, está obligado a la indemnización; sin perjuicio de la pena que le impongan las leyes por el delito o cuasidelito. (Art. 2.214)

El derecho de acción para una demanda civil amparada en este artículo nace de la sentencia condenatoria en materia penal, es decir, "el que ha cometido un delito o cuasi delito" se refiere a que debe existir la legalidad del acto, debe estar comprobada la ejecución del delito, a través de una sentencia en materia penal.

Por otra parte, impone al infractor o delincuente la obligación de la indemnización, esto es, el resarcimiento monetario en virtud del daño ocasionado. Dicho resarcimiento, en el caso de homicidios, puede recaer en los familiares de la víctima, conforme a ésta Ley.

\section{Conclusiones.}

En cualquier parte del mundo el enfermero o enfermera por el ejercicio de su función, lleva sobre sí una gran carga de responsabilidades, para con la profesión, con los pacientes, con sus colegas y con la sociedad en sí, por ello es importante que cada país tenga una definición o concepto claro de lo que significa este profesional, así como una delimitación de sus funciones, sobre todo porque su área de trabajo está conformada por un extenso equipo de salud, cuyas acciones comprometen las del otro y viceversa. 
Sidar E. Solórzano-Solórzano; Delia G. Bravo-Bonoso; Estrella M. Mero-Quijije; María E. Pincay-Cañarte; Dolores M. Cedeño-Holguín

La mala práctica del ejercicio de la función de la enfermería constituye un delito, lo cual representa el incumplimiento de la norma jurídica penal, y un daño para las partes del sistema de salud, principalmente el paciente.

En Ecuador, existe un conjunto de legislaciones que rigen tal función y en donde se establece tanto el deber ser o hacer del mencionado profesional de salud como sus sanciones o penas en caso de incumplimiento.

En la Carta Magna de la nación se encuentran bien fomentadas las bases para el desarrollo de toda la normativa jurídica relacionada con el ejercicio de la enfermería, representadas por dos derechos fundamentales: la vida y la salud, de allí se derivan una serie de leyes, códigos, resoluciones, jurisprudencias, entre otros documentos legales, que cubren las materias deontológicas, civiles, penales y administrativas relacionadas con las funciones de la enfermera (o).

Los casos de materia penal constituyen la base del desarrollo de acciones legales de otra materia, es decir, que una sentencia condenatoria en esta área representa el inicio de otras demandas, principalmente de tipo civil. En Ecuador, estas materias se encuentran representadas por el Código Civil y el Código Orgánico Integral Penal, incluso la materia especial está cubierta con la Ley Orgánica de Salud y la Ley de Ejercicio Profesional de las Enfermeras y Enfermeros del Ecuador.

En base al principio básico y universal del derecho que establece que la ignorancia de la Ley no excusa de su cumplimiento, es importante para el profesional de Enfermería ampliar sus conocimientos jurídicos con el fin de establecerse mayores niveles de exigencia en la práctica profesional, entablar una mejor relación con sus compañeros, con el paciente y con el entorno, con el propósito de evitar conflictos o denuncias. 
El enfermero o enfermera en el ejercicio de su función debe garantizar la idoneidad del servicio con el objeto de evitar demandas y al mismo tiempo, ayudar a proteger los derechos de sus pacientes.

\section{Bibliografía.}

Arias, F. (2012). El Proyecto de Investigación. Introducción a la metodología científica. (5ed.). Caracas, Venezuela: Episteme.

Carrara, F. (1991). Programa de Derecho Criminal (Vol. 1). Bogotá: Temis.

Código Civil de la República del Ecuador. (22 de mayo de 2016). www.justicia.gob.ec. Recuperado el 22 de junio de 2018, de https://www.justicia.gob.ec/wp-content/uploads/2018/02/08Codigo-Civil.pdf

Constitución de la República del Ecuador. (20 de octubre de 2008). http://www.inocar.mil.ec. Recuperado el 12 de junio de 2018, de http://www.inocar.mil.ec/web/images/lotaip/2015/literal_a/base_legal/A._Constitucion_repu blica_ecuador_2008constitucion.pdf

Definición. (s.f.). definicion.de. Recuperado el 22 de junio de 2018, de https://definicion.de/responsabilidad-civil/

Grisanti, A. (2005). Lecciones de Derecho Penal Parte General. Melvin.

Jiménez de Asúa, L. (1997). Lecciones de derecho penal. Harla.

Ley del Ejercicio Profesional de las Enfermeras y Enfermeros del Ecuador. (11 de febrero de 1998). leopoldolarrea.org. Recuperado el 13 de junio de 2018, de http://webcache.googleusercontent.com/search?q=cache: $1 \mathrm{psbxZgSdycJ}:$ leopoldolarrea.org/w p-content/uploads/2017/12/LEY-DE-EJERCICIO-PROFESIONAL-DE-LASENFERMERAS-Y-ENFERMEROS-DEL-ECUADOR.docx $+\& c d=3 \& \mathrm{hl}=\mathrm{es}-$ $419 \& \mathrm{ct}=\mathrm{clnk} \& \mathrm{gl}=\mathrm{ve}$

Ley Orgánica de Salud. (22 de enero de 2012). todaunavida.gob.ec. Recuperado el 13 de junio de 2018, de https://www.todaunavida.gob.ec/wp-content/uploads/downloads/2015/04/SALUDLEY_ORGANICA_DE_SALUD.pdf

Ministerio de Justicia, Derechos Humanos y Cultos de la República del Ecuador. (2014). Código Orgánico Integral Penal (Primera ed.). Quito, Ecuador: Subsecretaría de Desarrollo Normativo. Recuperado el 12 de junio de 2018, de https://www.justicia.gob.ec/wpcontent/uploads/2014/05/c\%C3\%B3digo_org\%C3\%A1nico_integral_penal_-_coip_ed._sdnmjdhc.pdf 
Sidar E. Solórzano-Solórzano; Delia G. Bravo-Bonoso; Estrella M. Mero-Quijije; María E. Pincay-Cañarte; Dolores M. Cedeño-Holguín

Ministerio de Salud Pública de la República del Ecuador. (2014). salud.gob.e. Recuperado el 12 de junio de 2018, de https://www.salud.gob.ec/msp-explica-el-art-146-del-coip/

Organización Mundial de la Salud. (1996). http://apps.who.int. Recuperado el 12 de junio de 2018, de

http://apps.who.int/iris/bitstream/handle/10665/41914/9243208608_spa.pdf?sequence=1\&is Allowed=y

Wolter Skluwer. (s.f.). guiasjuridicas.wolterskluwer.es. Recuperado el 22 de junio de 2018, de http://guiasjuridicas.wolterskluwer.es/Content/Documento.aspx?params=H4sIAAAAAAAE AMtMSbF1jTAAAUMzAzNLtbLUouLM_DxbIwMDC0NDIxOQQGZapUtckhlQaptWmJOcSoAnDJWLjUAAAA=WKE 